\title{
1. \\ Fragmenta Theoriae aequationum lineariter differentialium.
}

(Auctore C: J. D. Hill, math. prof. Lundae.)

C um aliquot calculi compendia, quae in hac elaboranda deteximus, in publicum praeire edenda credidimus, sequentia jam praemonenda putamus. Olim jam seriem $c f x+c_{1} \partial f x+c_{2} \partial^{2} f x+\ldots .+\ldots$ (tagmaticam jam nobis dictam) accuratius pensitavimus, et ejus summandae regulas in hoc ipso diario Tom. V. pag. 319 sq. descripsimus; unde facile vidisti, ipsam computatum iri, quoties coëfficientes $c, c_{1}, c_{2}$ etc. aliquatenus convergant, idque si vel functiones derivatae $\partial f x, \partial^{2} f x$ etc. minus notae (ut cum $f x=\Gamma x$ apud cel. Legendre), vel si hi coëfficientes variabiles essent. Cum haec observaverimus, de functione quacunque $\boldsymbol{X}$ in similem seriem evolvenda cura nobis fuit, et praecipue primum convergentiae obtinendae causa posuimus $f x=a_{0} \beta^{w_{0} \cdot x}+a_{1} \beta^{w_{1} \cdot x}+a_{2} \beta^{w_{2} \cdot x}+\ldots . .=\int\left(\boldsymbol{a} \beta^{w x}\right)$, exsistentibus ' $\boldsymbol{u}_{0}$, $w_{1}, w_{2}$ etc. quantitatibus parvis, et $a_{10}, a_{1}, a_{2}$ primum constantibus, tum vero variabilibus; deinde vero seriem antea descriptam casu generaliori, quo $c$, $c_{1}, c_{2}, \ldots$ functiones ipsius $x$ sunt, et $f x$ quaecunque, perscrutati sumus, utque functionis cujusvis $\boldsymbol{X}$ evolutionem consideravimus. Praecipuas huc pertinentes formulas, quivis facile reperiet. ${ }^{*}$ )

Ab altera vero parte, cum diu frustra solutionem aequationum lineariter differentialium quadraturae indefinitae ope instituendam perquisivimus, tandem persuasi fuimus, has suo ipsarum solvendi genere donandas esse, ideoque ipsarum indolem accuratius perscrutandam.

Data igitur ejusmodi aequatione lineari

$$
a_{0} y+a_{1} \partial y+\underset{x}{a_{2}} \partial^{2} y+\ldots+a_{x} \partial^{n} y=X,
$$

observavimus, partim in hac theoria partes, qualis haec sinistra $a_{0} y+\underset{x}{a_{1} \partial y+\ldots . .}$

*) Haud forsan importune tamen observare licet, si $f x$ sub forma $c+c_{1} x+$ $\frac{c_{2} x^{2}}{1.2}+\frac{c_{8} x^{3}}{1.2 .3}+\ldots$ evoluta fuerit, ita ad aequationes $c=\int a, c_{1}=\int a w, c_{2}=\int a w^{2}$, $c_{3}=\int a w^{3}$ etc. quarum solutionem l. c. dedimus, perventum iri.

Crelle's Journal f. d. M. Bd. XXV. Heft 1. 
est saepissime occurrere, ut neque calcules nostros breviter perfici neque theoremata concinne exprimi possent, nisi talem signo quodam simpliciori et expressivo (ex. gr. $(\stackrel{n}{a} \partial) y$ vel $\left.{ }_{a}^{n} \partial y\right)$ breviter indicaremus; partem substitutionem $y=u z$, quam et plurimi Geometrae ante nos sane instituerunt, singularem evolutiones legem introducere.

Posuimus igitur primum $a_{0} y+a_{1} \partial y+a_{2} \partial^{2} y+\ldots+a_{n} \partial^{n} y=(\stackrel{n}{a} \partial) y$, existente $\partial^{r} y=\frac{d^{r} y}{(d x)^{r}}$ et $\delta^{r} y$ seu $\partial_{c}^{r} y=\frac{\partial^{r} y^{\prime}}{1.2 .3 \ldots . . r}$, si $a_{0}, a_{1}, a_{2}$ etc. fnnctiones ipsius $x$ fuerint, - ut aequatio modo proposita breviter par $\imath^{n} \dot{\partial} y=$ $\boldsymbol{X}$ scribatur; deinde vero observavinus aliquid commodi attingi, si partim aequationem sub formam:

$$
a_{0} y+a_{1} \partial y+a_{2} \partial^{2} y+\ldots+a_{n} \partial^{n} y=X,
$$

eamque contractam per $\left(a^{n} \delta\right) y=X$ vel $(\stackrel{n}{n} \partial) y=X$ scriberemus, ubi $\delta^{r} y$ $=\partial_{c}^{r} y^{\prime}(\operatorname{apud}$ Arbogast $)=\frac{d^{r} y}{1.2 \ldots r(d x)^{r}}$, et partim si coëfficientes (variabiles, si placet) par $n_{i} ; n_{1}, n_{2}, \ldots$ signaremus, quo casu $n_{0} y+n_{1} \nabla y+n_{2} \delta^{2} y$. $+\ldots .+n_{n} \delta^{n} y$ brevius per $\left(n^{0} \delta\right) y$ vel $\left(n^{1} \delta\right) y$ indicatur, quo facto

$$
\left(n^{0} D\right) y=\boldsymbol{X}
$$

brevissime aequationem lineariter differentialem ordinis $n^{\text {ti }}$ indicat. Haec ad signa nostra intelligenda sufficiant.

Jan vero si substitutionem memoratam effeceris (vel in dissertatione cel. Libri in hoc Diario Tom. IX. divulgata inspexeris), posuerisque $(\stackrel{n}{a} \partial)(X y)$ $=b_{0} . y+b_{1}, \partial y+b_{2} . \partial^{2} y+\ldots .+b_{n} . \partial^{n} y$, videbis, coëfficientes $b_{0}, b_{1}, b_{2}$ etc. valoribus in fragmento mox subsequente exhibitis, gaudere.

Cum vero observavimus, terminum quemcunque ipsius $b_{1}$, ex. gr. $\boldsymbol{r} \boldsymbol{a}_{r} \partial^{r-1} \boldsymbol{X}$, ex correspondente ipsius $b_{0}, a_{r} \partial^{r} \boldsymbol{X}$, eodem modo oriri, ac si hunc secundum ipsum derivationis signum $\partial$ differentiaremus, (est nempe $\left.\frac{d\left(a_{r} \partial^{r}\right) X}{d(\partial)}=\frac{a_{r} d\left(\left(\partial^{r}, X\right)\right.}{d(\partial)}=a_{r} r \partial^{r-1} X\right)$, similiterque $b_{2}$ ex $b_{1}$ etc. oriri, perspeximus hujus seriei terminos singulari quodam derivationis genere a se invicem dependere, eoque siugulari signo distributiva $\mathcal{G}$ vel $\tau$ vel $\mathfrak{D}$, (cum naturale $\underset{d}{d}$ minus commode videtur) definiendo; de cujus indole jam sequens fragmentum fusius tractat. 


\section{Fragmentum I.}

Quoniam, ut jam indicavimus; formula tagmatica seu secundum derivata ipsius $y$ ordinata

$$
\boldsymbol{Y}=a_{0} y+a_{1} \partial y+a_{2} \partial^{2} y+\ldots+a_{n} \partial^{n} y
$$

quam et ita breviter scripsimus

$$
\dot{\boldsymbol{Y}}=(\stackrel{n}{\boldsymbol{a}} \partial) y
$$

ponendo $\boldsymbol{X} \boldsymbol{y}$ loco ipsius $y$, mutatur in novam formam linearem

$$
\boldsymbol{Y}^{\prime}=b_{0} y+b_{1} \partial y+b_{2} \partial^{2} y+\ldots+b_{n} \partial^{n} y
$$

seu brevius $\boldsymbol{Y}^{\prime}=\left(\boldsymbol{b}^{n} \partial\right) y$, existentibus

$$
\begin{aligned}
& \boldsymbol{b}_{1 \prime}=a_{0} \boldsymbol{X}+\boldsymbol{a}_{1} \partial \boldsymbol{X}+\boldsymbol{a}_{2} \partial^{2} \boldsymbol{X}+\ldots+\boldsymbol{a}_{n} \partial^{n} \boldsymbol{X}=(\boldsymbol{a} \partial) \boldsymbol{X} \\
& \boldsymbol{b}_{1}=\boldsymbol{a}_{1} \boldsymbol{X}+2 a_{2} \partial \boldsymbol{X}+3 a_{3} \partial^{2} \boldsymbol{X}+\ldots .+n a_{n} \partial^{n-1} \boldsymbol{X}=\vartheta((\boldsymbol{a} \partial) \boldsymbol{X}) \\
& \boldsymbol{b}_{2}=\boldsymbol{a}_{2} \boldsymbol{X}+3 a_{3} \partial \boldsymbol{X}+\ldots \ldots+n \frac{n-1}{2} a_{n} \partial^{n-2} \boldsymbol{X}=\frac{\boldsymbol{\vartheta}^{2}}{2}((\boldsymbol{a} \partial) \boldsymbol{X})
\end{aligned}
$$

generatimque $b_{r}=\frac{\vartheta^{r}}{1.2 \ldots r}\left(\left(a^{n} \partial\right) X\right)$; nemo non videt, formam $\left(b^{n} \dot{\partial}\right) y$ etiam hunc in modum explicari posse:

$$
\left(b^{n} \partial\right) y=b_{0} y+\vartheta b_{0} . \partial y+\frac{\vartheta^{2} b_{0}}{1.2} \cdot \partial^{2} y+\cdots \cdot+\frac{\vartheta^{n} b_{0}}{1.2 \ldots \ldots n} \cdot \partial^{n} y
$$

ubique scilicet $b_{0}$. loco formulae ipsi aequalis $(a \partial) X$ introducto. Similiter igitur et forma data $(\stackrel{n}{a} \partial) y$ per

vel etiam per

$$
a_{0} y+\vartheta a_{10} \cdot \partial y+\frac{1}{2} \vartheta^{2} a_{0} \cdot \partial^{2} y+\frac{1}{2.3} \vartheta^{3} a_{0} \cdot \partial^{3} y+\ldots
$$

aut per

$$
a y+\vartheta a \cdot \partial y+\vartheta^{2} a \cdot \partial y^{2}+\vartheta^{3} a \cdot \partial^{3} y+\ldots .+\vartheta^{n} a \cdot \partial^{n} y
$$

$$
\begin{gathered}
a_{0} y+\underset{c}{\vartheta_{c} a_{0}} \cdot \partial y+\underset{c}{\vartheta^{2}} a_{0} \cdot \partial^{2} y+\ldots, \\
\text { poneudo } a_{1}=\vartheta a_{0}, \boldsymbol{a}_{2}=\vartheta_{c}^{2} a_{0}=\frac{1}{2} \vartheta^{2} a_{0}, a_{3}=\underset{c}{\vartheta^{3} a_{0}}=\frac{1}{2.3} \cdot \vartheta^{3} a_{0} \text { etc. }
\end{gathered}
$$

explicatur; idemque de forma lineare quacunque, quandoquidem (a) y utcunque dari possit, tenendam rest.

Quoniam vero coëfficientes $a_{0}, a_{1}, a_{2}, \ldots a_{n}$, ad arbitrium accipi possunt, etiam haec signa $a, \vartheta a, \vartheta^{2} a, \vartheta^{r} a$ etc. similiterque exinde $b, \vartheta b^{\prime}$, $\vartheta^{r} b$ etc. functiones quascunque (ipsius $X_{0}$ si $y$ ipsius $x$ est funetio habenda, vel $\partial^{r} y \operatorname{loco}_{i} \frac{d^{r} y}{(d x)^{r}}$ scriptum est) significare possunt, easque diversissimas, 
1. Hill, Fragmenta Theoriac acquationum lineariter differentialium.

nulloque inter se vinculo junctas, praeterquam quod simul coëfficientes in eadem forma lineari sint. Haecque praecipua novi nostri calculi est vis, cum ita functiones arbitrariae innumerae in calculum simplicissimum introducantur.

Sin vero harum functionum prima $\boldsymbol{b}_{1}$ secundum functionis cujusdam $\boldsymbol{X}$ differentialia aliquo modo explicata datur, (id, quod varie efficitur in formis finitis, quandoquidem coëfficientes $a_{0}, a_{1}, a_{2}, \ldots a_{r}, \ldots a_{n}$ vel $a, \vartheta a, \vartheta^{2} a, \ldots$ arbitrariae sint functiones, numeroque finito $n$ adsint, scilicet ita: $b_{0}=$ (a) $\stackrel{n}{ } \boldsymbol{X}$, seu

$$
\boldsymbol{b}_{0}=a_{1} \boldsymbol{X}+a_{1} \partial \boldsymbol{X}+a_{2} \partial^{2} \boldsymbol{X}+\ldots+a_{n} \cdot \partial^{n} \boldsymbol{X}
$$

seu

$$
\boldsymbol{b}=\boldsymbol{a} \cdot \boldsymbol{X}+\vartheta^{a} \cdot \boldsymbol{\delta} \boldsymbol{X}+\vartheta^{2} a \cdot \delta^{2} \boldsymbol{X}+\ldots .+\vartheta^{n} a \cdot \delta^{n} \boldsymbol{X}
$$

tum etiam reliquarum explicandi modus datur, nempe

$$
\begin{gathered}
\vartheta \dot{b}=a_{1} \boldsymbol{X}+2 a_{2} \partial \boldsymbol{X}+\ldots+n a_{n} \partial^{n-1} \boldsymbol{X}, \\
\left(\operatorname{seu}=\vartheta a \cdot \boldsymbol{X}+\vartheta^{2} a \cdot \partial \boldsymbol{X}+\ldots+\frac{\vartheta^{n} a}{1.2 \ldots(n-1)} \cdot \partial^{n-1} \boldsymbol{X}\right),
\end{gathered}
$$

seu brevius $\vartheta b=\vartheta\left(\left(a^{n} \partial\right) X\right)$; similiterque

$$
\vartheta^{2} b=\vartheta(\vartheta b)=2 \cdot\left(a_{2} X+3 a_{3} \partial X+\ldots .+n_{2} \cdot a_{n} \partial^{n-2} \boldsymbol{X}\right)
$$

seu $\vartheta^{2} b=\vartheta^{2}((\dot{a} \partial) X)$, etc.

Patet vero $\vartheta \boldsymbol{b}$ esse formam linearem secundum derivata ipsius $\boldsymbol{X}$ uno gradu inferiórem $a c b$; quare et ipsam bunc in modum scribere licet: $\vartheta b=\left({ }^{\prime} a^{n-1} \partial\right) X$, existente ' $a_{0}=a_{1}=\vartheta a_{0}, \vartheta^{\prime} a_{0}=2 a_{2}=\vartheta^{2} a_{0}, \vartheta^{2}\left({ }^{\prime} a_{0}\right)=$ $2.3 a_{3}=\vartheta^{3} a$ generatimque $\vartheta^{r}\left({ }^{\prime} a\right)=\vartheta^{r+1} a$, seu $\vartheta^{r}(\vartheta a)=\vartheta^{r+1} a$, unde iterando elicitur $\vartheta^{r+n} a=\vartheta^{r} \vartheta^{n} a$.

Est scilicet $\vartheta b$ tum $=b_{1}=a_{1} X+2 a_{2} \partial X+3 a_{3} \partial^{2} X+\ldots+n a_{n} \partial^{n-1} X$ tum (ex modo positis) $={ }^{\prime} \boldsymbol{a} \boldsymbol{X}+\vartheta^{\prime} \boldsymbol{a} . \partial \boldsymbol{X}+\frac{1}{2} \vartheta^{\prime}{ }^{\prime} a . \partial^{2} \boldsymbol{X}+\ldots \ldots \ldots$ $+\frac{1}{2.3 \ldots(n-1)} \vartheta^{n-1}\left({ }^{\prime} a\right) \cdot \partial^{n-1} X$, itemque

$$
b_{1}=\vartheta a \cdot X+2 \cdot \frac{\vartheta^{2} a}{2} \cdot \partial X_{1}+3 \cdot \frac{\vartheta^{3} a}{2 \cdot 3} \cdot \partial^{2} X+\frac{+n \cdot \vartheta^{n} a}{1.2 \ldots . . n} \cdot \partial^{n-1} X,
$$

unde, coëfficientes ipsius $\partial^{r} \boldsymbol{X}$ comparando, dictae formulae elucent. Evolvendo vero $(\stackrel{n}{a})(\boldsymbol{X} \boldsymbol{y})+\left({ }^{n} \boldsymbol{\partial}\right)(\boldsymbol{X} \boldsymbol{y})$ facile evineitur, fore

seu

$$
\begin{aligned}
\vartheta^{m}\left(\left(a^{n} \partial\right) \boldsymbol{X}+\left(^{n} \partial\right) \boldsymbol{X}\right) & =\vartheta^{m}\left(a^{n} \partial\right) \boldsymbol{X}+\vartheta^{m}\left(c^{n} \partial\right) \boldsymbol{X}, \\
\vartheta^{m}(\alpha+\gamma) & =\vartheta^{m} \alpha+\vartheta^{m} \gamma .
\end{aligned}
$$

Quoniam vero. $\vartheta^{r}(\vartheta a)=\vartheta^{r+1} a$, quod et $=\vartheta^{r}\left({ }^{\prime} a\right)$, generatim, idque concinnius, $\vartheta$ a loco ' $a$ ponere licet; quare $\vartheta b=\left(\left(\vartheta^{n-1} a\right) \partial\right) X$, siquidem 
$b=(\stackrel{n}{\partial} \partial) X$ fuerit. Est igitur $\mathscr{\vartheta}\left(\left(a^{n} \partial\right) X\right)=\left(\left(\vartheta^{n-1} a\right) \partial\right) X$. Similiterque evincitur, esse $\vartheta^{2} b=\vartheta^{2}\left(\left(\boldsymbol{a}^{n} \partial\right) \boldsymbol{X}\right)=\left(\left(\vartheta^{2^{n}} a^{2}\right) \partial\right) \boldsymbol{X}$, generatimque $\vartheta^{r} b=\vartheta^{r}\left(\left(\boldsymbol{a}^{n} \partial \boldsymbol{X}\right)\right.$ $=\left(\left(\vartheta^{r} \stackrel{n-r}{a}\right) \partial\right) \boldsymbol{X}$.

Est enim

indeque

$$
\begin{aligned}
\boldsymbol{b} & =\boldsymbol{a} \cdot \boldsymbol{X}+\vartheta a \cdot \partial \boldsymbol{X}+\frac{\vartheta^{2} a}{1.2} \cdot \partial^{2} \boldsymbol{X}+\ldots+\frac{\vartheta^{r} a}{1.2 \ldots r} \partial^{r} \boldsymbol{X} \\
& +\frac{+\vartheta^{r+1} a}{1.2 \ldots r \cdot r+1} \cdot \partial^{r+1} \boldsymbol{X}+\ldots . .+\frac{\vartheta^{r+s} a}{1.2 \ldots(r+s)} \cdot \partial^{r+s} \boldsymbol{X}+\ldots
\end{aligned}
$$

atque

$$
\vartheta^{2} b=\vartheta^{2} a \cdot X+\ldots+\frac{r \cdot(r-1) \cdot \vartheta^{r}}{1.2 \ldots(r-2) \cdot(r-1) \cdot r} \cdot \partial^{r-2} X+\ldots
$$

atque

$\vartheta^{r} b=\frac{r_{1}^{r} \cdot \vartheta^{r} a}{1.2 \ldots . r} \cdot X+\frac{(r+1)_{1}^{r} \cdot \vartheta^{r+1}}{1.2 \ldots . r \cdot(r+1)} a \cdot \partial X+\ldots .+\frac{(r+s)_{1}^{r} \cdot \vartheta^{r+s} a \cdot \partial^{\cdot} \cdot X+\ldots . .}{1.2 \ldots . . .(s+1) \ldots(r+s)}$ existente $n_{1}^{r}=n(n-1) \ldots(n-(r-1))=$ facultate $r$ factorum arithmetice decrescentium $n, n-1, n-2$ etc., seu

$$
\vartheta^{2} b=\vartheta^{2} a \cdot X+\ldots+\vartheta^{r} a \cdot \frac{\partial^{r-2} X}{1.2 \ldots(r-2)}
$$

seu concinnius

$$
\vartheta^{r} b=\vartheta^{r} a \cdot X+\vartheta^{r+1} u \cdot \partial X+\ldots .+\frac{\vartheta^{r+s} a . \partial^{s} X+\ldots}{1.2 \ldots s},
$$

atque

$$
\vartheta^{2} b=\vartheta^{2} a \cdot X+\ldots+\vartheta^{r} a \cdot \delta^{r-2} X+\ldots .
$$

At

$$
\vartheta^{r} \boldsymbol{b}=\vartheta^{r} \boldsymbol{a} \cdot \boldsymbol{X}+\ldots .+\vartheta^{r+s} a \cdot \nu^{s} \boldsymbol{X}+\ldots .=\vartheta^{r}\left(a^{n} \partial \boldsymbol{X}\right) .
$$
atque

$$
\left(\left(\vartheta^{2} a\right) \partial\right) \boldsymbol{X}=\left(\vartheta^{2} a\right) \cdot \boldsymbol{X}+\vartheta^{n}\left(\vartheta^{2} a\right) \cdot \delta \boldsymbol{X}+\ldots .+\vartheta^{m}\left(\vartheta^{2} a\right) \cdot \delta^{m} \boldsymbol{X}+\ldots
$$

$$
\left(\left(\vartheta^{r} \stackrel{m}{a}^{\prime} \partial\right) \boldsymbol{X}=\left(\vartheta^{r} \boldsymbol{a}\right) \cdot \boldsymbol{X}+\vartheta\left(\vartheta^{r} \boldsymbol{a}\right) \cdot \delta \boldsymbol{X}+\ldots .+\vartheta^{s}\left(\vartheta^{r} \boldsymbol{a}\right) \cdot \delta^{s} \boldsymbol{X}+\ldots\right.
$$

praeterea $\vartheta^{r+s} a=\vartheta^{s}\left(\vartheta^{r} a\right)$, speciatimque $\vartheta^{m}\left(\vartheta^{2} a\right)=\vartheta^{m+2} a=\vartheta^{r} a$, posito $m=r-2$; quare formarum $\vartheta^{r}\left(\left(a^{n} \partial\right) X\right)=\left(\left(\vartheta^{r} a^{n-r}\right) \partial\right) X$ convenientia bene sibi constat.

Simul vero perspicitur, divisores numericos optime differentialibus ipsius $X$ jungi, et quidem hunc in modum $\frac{\partial^{r} X}{1.2 \ldots . . r}=\delta r X$ (seu $=\partial^{r} X$, ut apud cel. Arbogast), sub hac enim forma aullum faciunt negotium. Quo facto theorema nostrum universale pro forma lineari ${ }^{n} \partial(X y)$ evolvenda ita sonat. 


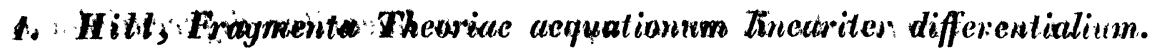

Existente $(\stackrel{n}{a} \partial) y=a y+\vartheta \mu \cdot \partial y+\vartheta^{2} a \cdot \partial^{2} y+\ldots+\vartheta^{n} a \cdot \partial^{n} y$, erit (a) $(\boldsymbol{X} \boldsymbol{y})=(\boldsymbol{a} \partial) \boldsymbol{X} \cdot \boldsymbol{y}+\left(\left(\left(\vartheta^{n-1} \boldsymbol{a}\right) \partial\right) \boldsymbol{X}\right) \cdot \partial y+\left(\left(\left(\vartheta^{2} a^{n-2}\right) \partial\right) \boldsymbol{X}\right) \cdot \partial^{2} y+\ldots$ $+\left(\left(\vartheta^{r} \stackrel{n-r}{a}\right) \partial\right) \boldsymbol{X} \cdot \partial^{r} y+\cdots+\left(\left(\vartheta^{n} a^{n-n}\right) \partial\right) \boldsymbol{X} \cdot \partial^{n} y$

ubi

$$
\begin{gathered}
\left(\left(\vartheta^{r} a^{n-r}\right) \partial\right) \boldsymbol{X}=\vartheta^{r} \boldsymbol{a} \cdot \boldsymbol{X}+\vartheta^{r+1} \boldsymbol{a} \cdot \boldsymbol{\partial}+\vartheta^{r+2} a \cdot \delta^{2} \boldsymbol{X}+\vartheta^{r+s} a \cdot \vartheta^{s} \boldsymbol{X}+\ldots \\
\ldots+\vartheta^{n} a \cdot \diamond^{n-r} \boldsymbol{X}
\end{gathered}
$$

vel etiam, facto $\left(a^{n} \partial\right) \boldsymbol{X}=a$, erit $\left(\boldsymbol{a}^{n} \partial\right)(\boldsymbol{X} \boldsymbol{y})=\alpha y+\vartheta \alpha \cdot \partial y+\vartheta^{2} \alpha \cdot \partial^{2} y+\cdots$ $\ldots+\vartheta^{n} a . \partial_{c}^{n} \dot{y}$; atque $\left.\vartheta^{m} \alpha=\vartheta^{m}(\stackrel{n}{a} \partial) \boldsymbol{X}=\left(\left(\vartheta^{m} a^{n}\right) \partial\right) \boldsymbol{X} . *^{*}\right)$ Hac igilur forma adhibita, valor transformati polynomii tagmatici omnimodi explicitus, ideoque indepedenter ut dicunt, exhibebitur, nempe in coëfficientibus datis $a, \vartheta a, \vartheta^{2} a$ etc. expressus.

Forma vero $\left(a^{n} \partial\right)(\boldsymbol{X} y)=\left(b^{n} \partial\right) y$ seu $=\left(\left(\left(\boldsymbol{a}^{n} \partial\right) \boldsymbol{X}^{n}\right) \partial\right) y$. est implicita, casus evolutio inchoata haec est:

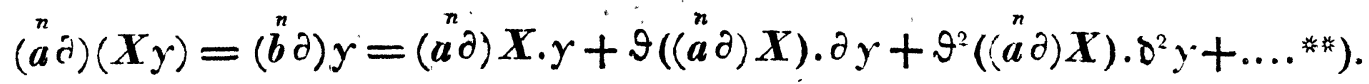

*) Formula haec facile ope notissima

$$
\partial_{c}^{r}(\boldsymbol{X} \boldsymbol{y})=\underset{c}{\partial^{r}} \boldsymbol{X} \cdot \boldsymbol{y}+\underset{c}{\partial^{r-1}} \boldsymbol{X} . \partial y+\underset{c}{\partial^{r-2}} \boldsymbol{X} \cdot \partial^{2} y+\ldots
$$

domonstratur. Ita énim $(a \partial)(X y)$ seu polynomium

$$
\boldsymbol{a} . \boldsymbol{X} y+\boldsymbol{\vartheta} a . \partial(\boldsymbol{X} y)+\boldsymbol{\vartheta}^{2} \underset{c}{a . \partial^{2}}(\boldsymbol{X} y)+\ldots .+\vartheta^{r} \boldsymbol{a} \cdot \partial_{c}^{r}\left(X_{y^{\prime}}\right)+\ldots
$$

in hoc

ideoque in

$$
\begin{aligned}
& \boldsymbol{a} \cdot \boldsymbol{X} \boldsymbol{y}+\boldsymbol{\theta} \boldsymbol{a} \cdot(\partial \boldsymbol{X} \cdot \boldsymbol{y}+\boldsymbol{X} \cdot \partial \boldsymbol{y}) \\
& +\vartheta^{2} \cdot a \cdot\left(\partial^{2} X \cdot y+\partial X \cdot \partial y+\boldsymbol{X} \cdot \partial_{c}^{2} y\right) \\
& +\vartheta^{3} a \cdot\left(\partial^{3} X \cdot y+\partial_{c}^{2} X \cdot \partial y+\partial X \cdot \partial_{c}^{2} y+X \cdot \partial_{c}^{3} y\right) \\
& \text { t. . . . . . . . . . . } \\
& +\vartheta^{r} a \cdot\left(\partial^{r} \boldsymbol{X} y+\partial_{c}^{r-1} \boldsymbol{X} . \partial_{\boldsymbol{c}} y+\partial^{r-2} \boldsymbol{X} \cdot \partial^{2} y+\ldots\right)
\end{aligned}
$$

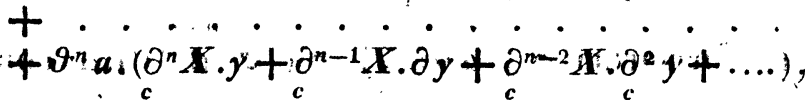

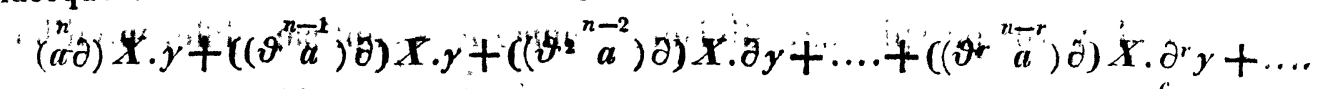

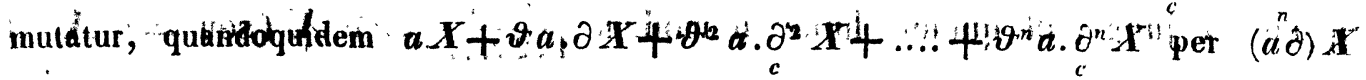

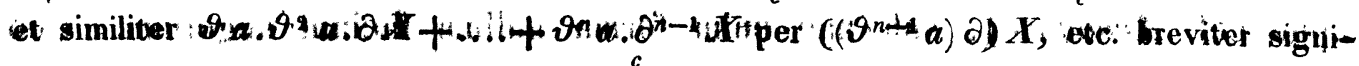
fipatur.i.

*) Existente igitur $\alpha=(a \partial) X$; quantitates $\vartheta \alpha, \vartheta^{2} \alpha, \ldots \vartheta^{\prime 2} \alpha$ caëflicien- 
Praeterea monemus; signum nostrum $\vartheta$ vel $\mathfrak{D}$, originarie hoc valebat, ut loco ipsius a. $\partial^{r} y$, si huic praefigebatur, sumeretur $\boldsymbol{r a} \partial^{r-1} y$, nempe $\vartheta\left(a . \partial^{r} y\right)=$ $\boldsymbol{r} a \partial^{r-1} y$, existentibns $a$ et $y$ quidem functionibus ipsius, $x$, mamente tamen $a$ secundum operationem $\vartheta$ constante (quare et $\vartheta_{y}\left(a \partial^{r} y\right.$ ) scribi potest), praeterea, ut jam innumus, distributivuin esse (seu ejus indolis, ut $\vartheta(p+q)$ $=\vartheta p+\vartheta q$ sit, quoties $p$ et $q$ series tagmaticae fuerint), exque hac ipsa modo instituta evolutione novum nancisci significatum. Videmus eoim, tagma $\boldsymbol{b}=(\stackrel{n}{a} \partial) \boldsymbol{X}$ huic opérationi $(\vartheta)$ subjectum ex forma sua explicata

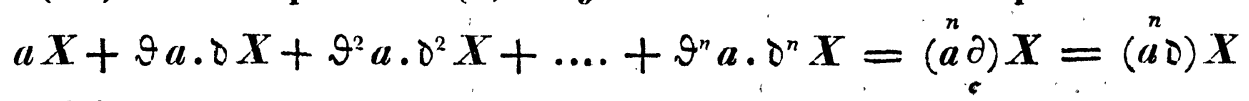

migrari in

$$
\vartheta a \cdot X+\vartheta^{2} a \cdot \partial \boldsymbol{X}+\vartheta^{3} a \cdot \delta^{2} \boldsymbol{X}+\ldots .+\vartheta^{r+3} a \cdot \vartheta^{r} \boldsymbol{X}+\ldots . .+\vartheta^{n} a \cdot \vartheta^{n-3} \boldsymbol{X} \text {, }
$$

(est enim $\left.\vartheta\left(\delta^{r} y\right)=\vartheta \frac{\partial^{r} y}{1.2 \ldots r r}=\frac{r \partial^{r+1} y^{\prime}}{1.2 \ldots(r-1) r}=\frac{\partial^{r-1} y^{\prime}}{1.2 \ldots(r-1)}=\delta^{r-1} y\right)$, ideoque $\vartheta((\stackrel{n}{a} \partial) X)$ significare, non modo in unoquoqũe ipsius $(\stackrel{n}{a} \partial) X$ explicati termino $\left(\vartheta^{r+1} a \cdot \delta^{r-1} \boldsymbol{X}\right)$ esse sumendum $\delta^{r} \boldsymbol{X}$ loco ipsius $\delta^{r+1} \overline{\boldsymbol{X}}$ ( quo facto in $\vartheta^{r+1} a \cdot \delta^{r} \boldsymbol{X}$ migrat), sed etiam, si mavis, mutandum esse $\vartheta^{r} a$ in $\vartheta^{r+1} a$, existente $\vartheta^{n+1} a=0$ (utat haudquaquam praesente). Ut igitur totam novi

tes derivatarum $\partial y, \delta^{2} y, \ldots \delta^{r} y$ ipsius $y$ in serie tagmatice evoluta $(\stackrel{n}{a} \partial)(X y)$ significant. Etiam ex hac notione ipsarum proprietas fundamentalis $\vartheta^{v} \vartheta^{n} \alpha=\vartheta^{\nu+m} \alpha$ ita demonstrari potest. Suscipiamus scilicet evolutionem formulae $(a \partial)(\boldsymbol{X} y \boldsymbol{Z})$ idque dupliciter, faciendo mado $\boldsymbol{X} \boldsymbol{Z}=\boldsymbol{X} \cdot(\boldsymbol{Z} \boldsymbol{Z})$ et modo $=(\boldsymbol{X Z}) \cdot y$. Illo casu erit, si $\alpha-(\stackrel{n}{a} \partial) \boldsymbol{X}$ valor aliquatenus-evolutus hoc vero $=\alpha \cdot y \boldsymbol{Z}+\boldsymbol{\vartheta} \boldsymbol{\alpha} \cdot \partial(y \boldsymbol{Z})+\boldsymbol{\vartheta}^{2} \alpha \cdot \partial_{c}^{2}(y \boldsymbol{Z})+\ldots .+\boldsymbol{\vartheta}^{r} \boldsymbol{\alpha} \cdot \partial^{r}(y \boldsymbol{Z})+\ldots$

$$
=(\stackrel{n}{a} \partial)(\boldsymbol{X} \boldsymbol{Z}) \cdot \boldsymbol{y}+\boldsymbol{\vartheta}(\stackrel{n}{\boldsymbol{a}} \partial)(\boldsymbol{X} \boldsymbol{Z}) \cdot \partial \boldsymbol{y}+\ldots . .+\boldsymbol{\vartheta}^{v}(\stackrel{n}{\boldsymbol{a}} \partial)(\boldsymbol{X} \boldsymbol{Z}) \cdot \partial^{v} \boldsymbol{y}+\ldots
$$

Ut vero hi valores comparari possint, ulterius evolvendum est tum $\partial^{r}(y Z)$ tum $\vartheta^{n}(\stackrel{n}{a} \partial)(\boldsymbol{X} \boldsymbol{Z})$.

Est vero $\partial_{c}^{r}(y \boldsymbol{Z})=\boldsymbol{Z} \cdot \partial_{c}^{r} y+\partial \boldsymbol{Z} \cdot \partial_{c}^{r-1} y+\ldots .+\partial_{c}^{m} \boldsymbol{Z} . \partial_{c}^{r-m} y+\ldots+\ldots$, alque

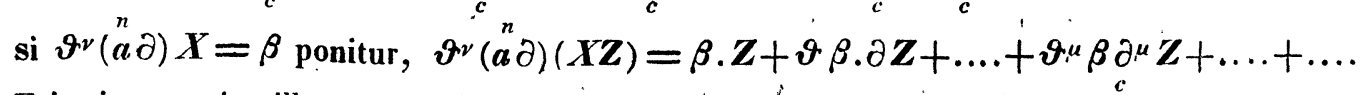
Erit itaque in illo casu terminus generalis $\doteq \vartheta^{r} \alpha \cdot \partial^{m} Z \cdot \partial^{r-m} y^{c}$ atque in hoc $=\vartheta^{\mu} \beta . \partial^{\mu} \boldsymbol{Z} . \partial_{c}^{\nu} y^{\prime}$, qui utrique necessario conveniant, si $m=\mu$ atque $\nu=r-m$ seu $r=\nu+m$ facitur. Inde igitur colligitur $\boldsymbol{\vartheta}^{\prime m} \beta=\vartheta^{\prime} \alpha=\vartheta^{v+m} \alpha$. Est vero $\beta=\vartheta^{v} \alpha$, ideoque $\vartheta^{\cdot v+m} \alpha=\vartheta^{m}\left(\vartheta^{v} \alpha\right), Q$. E. D.

Ex ipsa hac demonstratione vero patet, $\vartheta$ ad derivata functionis $\boldsymbol{Y}$ referri, secundum quae tum $\alpha$ tum $\beta$ ordinata sunt. 
1. Hill, Fragmenta Theoriae aequationum lineariter differentialium.

nostri calculi vim et indolem breviter teneas; ponamus $X=f a$ atque $f(x+e)$ $=f x+e f_{1} x+e^{2} f_{2} x+\ldots .+e^{r} f_{r} x+\ldots .$, seu $\delta^{r} X=f_{r} x$, ut habeamus functionem derivatarum seriem $f x, f_{1} x, f_{2} x, \ldots f_{r} x, f_{n} x$, quarum prima tantum utcunque sumi potest, reliquae vero determiuato modo; nempe notissimo derivandi, inde successive generantur; adsumamusque praeterea aequalem $n+1$ functionum omnino arbitrariarum numerum, "quae tamen certo ordine sibi invicem subsequuntur, ideoque, ut ipse hic ordo recte tenealur, per $a$, $\vartheta a, \vartheta^{2} a, \ldots . \vartheta^{r} a, \ldots$. indicantur ; et functiones utriusque seriei ejusdem loci in se invicem ducamus productaque in unam summam colligamus

$$
a f x+\vartheta a \cdot f_{1} x+\vartheta^{2} a \cdot f_{2} x+\ldots .+\vartheta^{r} a \cdot f_{r} x+\ldots \ldots+\vartheta^{n} a \cdot f_{n} x(=\sigma),
$$

quam breviter' signo $\left(a^{n} \partial\right) f x$ atque nomine seriei tagmaticae ordinis $n$ insignimus. His positis, series tagmatica in similem mutatur, vel functionem quamcunque derivativam $\left(f_{r} x\right)$ in proxime antecedentem $\left(f_{r-1} x\right)$ mutando, id quod per $\vartheta_{f}$ insignitur, ut $\vartheta_{f}\left(f_{r} x\right)=f_{r-1} x$ sit, atque

$$
\vartheta_{f}\left(\vartheta^{r} a \cdot f_{r} x+\vartheta^{n} f_{n} x\right)=\vartheta^{r} a \cdot f_{r-1} x+\vartheta^{n} a \cdot f_{n-1} x,
$$

vel etiam unamquamque functionem arbitrariam $\left(\vartheta^{r} a\right)$ in ipsam proximo ordine subsequentem $\left(\vartheta^{r+1} a\right)$ mutando, id quod per $\vartheta_{a}$ indicari potest, ut $\vartheta_{a}\left(\vartheta^{r} a\right)=\vartheta^{r+1} a$ fit, atque

$$
\vartheta_{a}\left(\vartheta^{r} a \cdot f_{r} x+\vartheta^{n} a \cdot f_{n} x\right)=\vartheta^{r+1} a \cdot f_{r} x+\vartheta^{n+1} a \cdot f_{n} x ;
$$

utroque vero modo idem obtinebitur, nempe $\vartheta a \cdot f x+\vartheta^{2} a \cdot f_{1} x+\ldots .+\vartheta^{r} a \cdot f_{r-1} x+\vartheta^{r+1} a \cdot f_{r} x+\ldots .+\vartheta^{n} a \cdot f_{n-1} x$, quod ipsius $(\stackrel{n}{a} \dot{\partial}) f x$ tagma primum dicimus et per $\dot{\vartheta}((\stackrel{n}{a} \dot{\partial}) f x)$ insignimus. Est igitur hoc et $=\vartheta_{f}\left(\left(\boldsymbol{a}^{n} \partial\right) f(x)\right)$ et $=\vartheta_{a}\left(\left(\boldsymbol{a}^{n} \partial\right) f(x)\right)$; nam obiter determinatum tantum numerum $(n+1)$ tum functionum derivatarum $f x\left(=\partial^{0} f x\right)$, $f_{1} x, \ldots f_{n} x$, tum arbitrarium $a, \vartheta a, \vartheta^{2} a, \ldots \vartheta^{n} a$ consideramus, quare tum $\vartheta_{f} f x=0$, tum $\vartheta_{a}\left(\vartheta^{n} a\right)=0$, cum $\vartheta^{n+1} a$ haud detur.

Patet vero similiter tagma secundum

$$
\vartheta^{2}((\stackrel{n}{a} \partial) f x) \text { per } \vartheta(\vartheta((\stackrel{n}{\partial} \partial) f x))
$$

et tertium

$$
\vartheta^{3}\left(\left(a^{n} \partial\right) f x\right) \text { per } \vartheta\left(\vartheta^{2}\left(\left(a^{n} \partial\right) f x\right)\right) \text { etc. }
$$

generatimque $\vartheta^{r}\left(a^{n} \partial\right) f x$ per $\vartheta\left(\vartheta^{r-1}\left(a^{n} \partial\right) f x\right)$ definitum iri, horunque utrumque duplicem significatum specialem tribui posse, nempe $\vartheta_{f}^{r} \sigma$ et $\vartheta_{a}^{r} \sigma$, seu 
1. Hill, Fragmenta Theoriae aequationum lineariter differentialium.

ejusmodi, ut vel ad mutationem functionum derivatarum vel ad arbitrariarum referatur, quod utrumque eodem redit. *)

Praeterea hujusmodi signa proprie non nisi seriei tagmaticae ay+ $\vartheta a . \delta y+\ldots .+\vartheta^{n} a \cdot \delta^{n} y$ vel ejus signo complexo $\left(a^{n} \delta\right) y$ praefigi potest, improprie vero signo functionis, ex. gr. $\vartheta \boldsymbol{F} x$, quo casu significatu carebit, nisi haec functio in ejusmodi seriem applicata fuerit. Ex. gr. si $F x=(\stackrel{n}{a} \partial) f x$ fuerit, sane loco ipsius $\mathcal{\vartheta}^{r}\left(a^{n} \partial\right) f x$ breviter $\mathcal{\vartheta}^{r} \boldsymbol{H}^{\prime} x$ scribere licebit.

Forma vero $(\stackrel{n}{a} \delta)$ y novum quasi functionum genus constituit, cujus evolutioni tagmata nostra aeque inserviunt ac vulgaribus differentialia, quodque ab his sejungi nequit, utrisque ad idem doctrinae corpus pertinentibus, et series $(\stackrel{n}{a} \delta)(X y)=(\stackrel{n}{a} \delta) X \cdot y+\vartheta\left(\left(a^{n} \delta\right) X\right) . D y+\frac{1}{2} \vartheta^{2}\left(\left(a_{a}^{n} \delta\right) X\right) \delta^{2} y+\cdots$ seu, si $n=\infty$ fuerit, (qui casus singulari cautione tractandus est), ideoque $n$ seu $\infty$ non adscribitur, haec

$$
(\hat{a} \downarrow)(\boldsymbol{X} y)=\hat{a} \delta X \cdot y+\frac{1}{2} \vartheta(\hat{a} \delta X) . \delta y+\frac{1}{2.3} \vartheta^{2}(\hat{a} \delta X) \delta^{2} y+\ldots
$$

his formis idem est ac series Taylorea vulgaribus.

Patet vero formas similes plurium arbitrarium dari, quae considerando aequationem differentialem lineariter particularem

$$
\left.\begin{array}{r}
a_{1} z+a_{x}^{\prime} \partial z+a_{x}^{\prime \prime} b_{x}^{2} z+\ldots . \\
+a_{y} \partial z+a_{1}^{\prime} b_{x y} \partial+\ldots \\
+a_{\prime \prime} b_{2}^{2} z+\ldots .
\end{array}\right\}=\mathbf{Z}, \text { facile inveniuntur, }
$$

de quibus tamen alio tempore. In praesente vero exemplum tantum unum alterumve usum calculi nostri monstrans, subjungemus.

\section{Fragmentum II.}

Probl. Functionibus quibusdam $(n)$ datis videlicet $b_{0}, b_{1}, b_{2}, \ldots$ $b_{r}, \ldots b_{n}$, insuperque aliqua $X$, alias totidem $a_{0}, a_{1}, a_{2}, \ldots a_{n}$ ita definire, ut ipsae coëfficientes ad hujus $(X)$ differentialia sint, dum lineariter ad has explicantur; nempe

$$
\boldsymbol{b}_{0}=(\stackrel{n}{a} \partial) \boldsymbol{X}, \quad \boldsymbol{b}_{1}=\vartheta(\stackrel{n}{a} \partial) \boldsymbol{X}, \quad \boldsymbol{b}_{2}=\frac{\vartheta^{2}}{2}(\stackrel{n}{a} \partial) X, \text { etc. }
$$

*) Operationis hujus tagmaticae regulae, quales jam in textu demonstrantur, breviter in programmate d. 22. Jun. 1835 dato indicata sunt. (Additamentum editoris.)

Crelle's Journal f. d. M. Bd. XXV. Heft 1. 
10 1. Hill, Fragmenta Theoriae aequationum lineariter difierentialium.

Functio vero $\boldsymbol{X}$ quaecunque esse potest, dummodo si algebraica integra fuerit, gradus saltem altioris ac $(n)$. Quae cum ita desiderentur, erit generatim $b_{r}=\frac{\boldsymbol{\vartheta}^{r}}{1.2 \ldots . . r}\left(a^{2}\right) X$, seu explicatione rite instituta $b_{r}=a_{r} \boldsymbol{X}+(r+1)_{1} \cdot a_{r+1} \partial \boldsymbol{X}+\ldots .+(r+s)_{s}^{a_{r+s}} \partial^{r} \boldsymbol{X}+\ldots .+n_{n-r} a_{n} \partial^{n-r} \boldsymbol{X}$, ideoque speciatim

quandoquidem $a_{n+s}=0$,

$$
\text { (1) } b_{n}=a_{n} X \text {, }
$$

(2) $b_{n-1}=a_{n-1} X+n a_{n} \partial X$,

(3) $b_{n-2}=a_{n-2} X+(n-1) a_{n-1} \partial X+n_{2} a_{n} \partial^{2} X$,

(4) ${ }^{\prime} b_{n-3}=a_{n-3} X+(n-2) a_{n-2} \partial X+(n-1)_{2} a_{n-1} \partial^{2} X+n_{3} a_{n} \partial^{3} X$, generatimque

$$
\begin{aligned}
\boldsymbol{b}_{n-s}=a_{n-s} \boldsymbol{X} & +(n-(s-1)) \cdot a_{n-(s-1)} \cdot \partial \boldsymbol{X} \\
& +(n-(s-2))_{2} \cdot a_{n-(s-2)} \cdot \partial^{2} \boldsymbol{X}+\ldots .+n_{s} a_{n} \partial^{s} \boldsymbol{X},
\end{aligned}
$$

quas aequationes et retro juxta regulam $\frac{\vartheta b_{r}}{r+1}=b_{r+1}$, seu

$$
\vartheta b_{n-1}=n b_{n}, \quad \vartheta b_{n-2}=(n-1) b_{n-1}, \quad \vartheta b_{n-3}=(n-2) b_{n-2} \text {, etc. }
$$

comprobare possis. Harum vera aequationum prima (1) aperte praebet $a_{n}=\frac{b_{n}}{X}$, hocque valore in subsidium vocato, secunda (2) suppeditat

$$
a_{n-1}=\frac{b_{n-1}}{X}-\frac{n \cdot b_{n} \partial X}{X^{2}}
$$

similiterque deinde ex tertia (3) habetur

$$
a_{n-2}=\frac{b_{n-2}}{X}-\frac{(n-1) b_{n-1} \partial X}{X^{2}}+n_{2} b_{n}\left(\frac{2(\partial X)^{2}}{X^{3}}-\frac{\partial^{2} X}{X^{2}}\right)
$$

item ex sequente habebitur $a_{n-3}$, et ex reliquis successive $a_{n-4}, a_{n-3}$ etc. usque ad $a_{0}$, quae ultima ex aequatione $b_{0}=(\stackrel{n}{a} \partial) X=a_{0} X+a_{1} \partial X+\ldots$ obtinetur.

Quoniam enim unaquaeque aequatio posterior non nisi unam habet ignotam, quae iis, quae jam ex prioribus quaerantur, accedit, has aequationes solvendi facultas per se constat. At vero et ipsae hae solutiones sub forma brevi memoratu digna exhiberi possunt. Patet enim, ex valoribus modo expositis fore

(1') $a_{n}=b_{n} X^{-1}$

(2') $a_{n-1}=b_{n-1} X^{-1}+n b_{n} \cdot \partial\left(X^{-1}\right)$;

(3') $a_{n-2}=b_{n-2} X^{-1}+(n-1) \cdot b_{n-1} \cdot \partial\left(X^{-1}\right)+n_{2} b_{n} \partial^{2}\left(X^{-1}\right)$; 
indeque jam conjicitur

$$
\begin{aligned}
a_{n-3}=b_{n-3} \cdot X^{-1}+(n-2) \cdot b_{n-2} \partial\left(X^{-1}\right) & +(n-1)_{2} \cdot b_{n-1} \cdot \partial^{2}\left(X^{-1}\right) \\
& +n_{3} b_{n} \partial^{3}\left(X^{-1}\right)
\end{aligned}
$$

id quod et facile comprobatur, hos valores $\left(1^{\prime}, 2^{\prime}, 3^{\prime}, 4^{\prime}\right)$ in aequationem (4) substituendo; ita enim haec evadit

$$
\text { (4) } b_{n-3}=b_{n-3} \cdot X \cdot X^{-1} \text {, }
$$

quandoquidem coëfficiens ipsius $b_{n-2}$ ita fit $(n-2) \cdot\left(X \partial X^{-1}+X^{-1} \partial X\right)$ seu $=(n-2) \partial \cdot\left(\boldsymbol{X} \cdot \boldsymbol{X}^{-1}\right)$, atque ipsius $b_{n-1}:(n-1)_{2}\left(\boldsymbol{X} \partial^{2}\left(\boldsymbol{X}^{-1}\right)+2 \partial \boldsymbol{X} \cdot \partial\left(\boldsymbol{X}^{-1}\right)\right.$ $\left.+\partial^{2} \boldsymbol{X} \cdot\left(\boldsymbol{X}^{-1}\right)\right)$ seu $(n-1)_{2} \cdot \partial^{2}\left(\boldsymbol{X} \cdot \boldsymbol{X}^{-1}\right)$, itemque ipsius $b_{n}$ :

$$
n_{3} \cdot\left[\boldsymbol{X}^{3}\left(\boldsymbol{X}^{-1}\right)+3 \cdot \partial \boldsymbol{X} \cdot \partial^{2}\left(\boldsymbol{X}^{-1}\right)+3 \partial^{2} \boldsymbol{X} \cdot \partial\left(\boldsymbol{X}^{-1}\right)+\partial^{3} \boldsymbol{X} \cdot\left(\boldsymbol{X}^{-1}\right)\right]
$$

seu $n_{3} \partial^{3}\left(X^{-1} X\right)$; quae omnes aperte nibilo aequantur, quandoquidem $\boldsymbol{X} . \boldsymbol{X}^{-1}$ $=1=$ const. sit, ideoque $\partial^{r}\left(\boldsymbol{X} \cdot \boldsymbol{X}^{-1}\right)=0$. Eodem vero modo positionis similis

$$
\begin{aligned}
a_{n-4}=b_{n-4} X^{-1} & +(n-3) b_{n-3} \partial X^{-1}+(n-2)_{2} b_{n-2} \partial^{2}\left(X^{-1}\right) \\
- & +(n-1)_{3} b_{n-1} \partial^{3}\left(X^{-1}\right)+n_{4} b_{n} \partial^{4}\left(X^{-1}\right)
\end{aligned}
$$

generaliorisque

$$
\begin{aligned}
a_{n-s}=b_{n-s} \cdot X^{-1} & +(n-(s-1)) b_{(n-(s-1))} \cdot \partial\left(\boldsymbol{X}^{-1}\right) \\
& +(n-(s-2))_{2} \cdot b_{(n-(s-2))} \cdot \partial^{2}\left(X^{-1}\right)+\ldots .+\ldots \\
& +(n-(s-r))_{r} \cdot b_{(n-(s-r))} \cdot \partial^{r}\left(X^{-1}\right)+\ldots
\end{aligned}
$$

veritas evincitur, hujusmodi valores in aequationem (5) vel 5 introducendo quae ita $\boldsymbol{b}_{n-m}$ cum coëfficiente evoluta, quae facile in $(n-m)_{s-m} . \partial^{s-m}\left(\boldsymbol{X} \cdot \boldsymbol{X}^{-1}\right)$ contrahitur, continere invenitur.

Omnes vero hae coëfficientes aperte nihilo aequantur, praeterquam cum $m=s$ fuerit, quo casu $=\partial^{0}\left(\boldsymbol{X} \cdot \boldsymbol{X}^{-1}\right)=\boldsymbol{X} \cdot \boldsymbol{X}^{-1}=1$ evadit. Aequatio igitur (5) haec $b_{n-s}=b_{n-s} .1$ fit, quae identica est. Posito igitur $Z=X^{-1}$, ex eis, quae modo demonstrabantur, erit

$$
a_{m}=b_{m} \cdot \boldsymbol{Z}+(m+1) b_{m+1} \partial \mathbf{Z}+(m+2)_{2} b_{m+2} \cdot \partial^{2} \boldsymbol{Z}+\ldots,
$$

speciatimque

$$
a_{0}=b_{0} \boldsymbol{Z}+b_{1} \partial \boldsymbol{Z}+b_{2} \partial^{2} \boldsymbol{Z}+\ldots+b_{n} \partial^{n} \boldsymbol{Z}
$$

atque

$$
a_{1}=b_{1} \mathbf{Z}+2 b_{2} \partial \mathbf{Z}+3 b_{3} \partial \mathbf{Z}+\ldots . . . .
$$

seu brevius $a_{0}=\left(b^{n} \partial\right) \boldsymbol{Z}$ atque $a_{1}=\vartheta\left(b^{n} \partial\right) \boldsymbol{Z}$, similiterque $a_{2}=\frac{\vartheta^{2}}{2}\left(b^{n} \partial \boldsymbol{Z}\right)$ $a_{3}=\frac{1}{2.3} \cdot \vartheta^{3}\left(b^{n} \partial Z\right)$ etc. Singulare igitur hinc emergit : 
Theorema. Si junctim fuerint

$b_{0}=(\stackrel{n}{a} \partial) X, \quad b_{1}=\vartheta(\stackrel{n}{a} \partial) X, \quad b_{2}=\frac{1}{2} \vartheta^{2}(a) X, \ldots, b_{r}=\frac{1}{1.2 \ldots r} \vartheta^{r} a^{n} \partial X, \ldots$

inverso ordine erant

$$
\ldots b_{n}=\frac{1}{1.2 \ldots . n} \vartheta^{n}(\stackrel{n}{a} \partial X)
$$

$$
\begin{aligned}
a_{0}=\left(b^{n} \partial\right) Z, \quad a_{1} & =\vartheta\left(b^{n} \partial\right) Z, \quad a_{2}=\frac{\vartheta^{2}}{2}\left(b^{n} \partial\right) Z, \ldots \text { gereratimque } \\
a_{r} & =\frac{1}{1.2 \ldots r}\left(\vartheta^{r}\left(b^{n} \partial\right) Z\right), \text { existente } \mathbf{Z}=\frac{1}{X} .
\end{aligned}
$$

Hujus Theorematis ope varia problemata simplicissime solvuntur. Ex. gr. Datis aequationibus

$$
\begin{array}{cl}
b_{0}=a_{0}+a_{1} e+a_{2} e^{2}+a_{3} e^{3}+ & =\boldsymbol{A}(e) \\
b_{1}=a_{1}+2 a_{2} e+3 a_{3} e^{2}+ & =\underset{e}{ } A(e) \\
b_{2}=a_{2}+a_{2}+3 a_{3} e+ & =\underset{e}{\delta^{2}} A(e) \\
\quad \text { etc. } &
\end{array}
$$

atque universim $b_{n}=\oslash^{n}(A(e))$, inversim coëfficientes $a_{0}, a_{1}, a_{2}, \ldots$ functionis $\boldsymbol{A}(\boldsymbol{e})$ invenire.

Ponendo $X=\beta^{e x}, \boldsymbol{Z}=\beta^{-e x}$, atque ad finem $x=0$, invenies prisnum $a_{0}=\left(b^{n} \partial\right) \beta^{-e x}=b_{0} \beta^{-e x}+b_{1} \partial \beta^{-e x}+b_{2} \partial^{2} \beta^{-e x}+\ldots$, seu $a_{0}=$ $b_{0}-b_{1} e+b_{2} e^{2}-\ldots$. , quae series $=B(e)$ ponitur, ut $B_{(e)}$ functio data ipsius $e$ sit, deinde $a_{1}=b_{1}-2 b_{2} e+\ldots$. seu $a_{1}=-\partial_{e}^{\partial B}(e)=-B_{1} e$, et

et atque igitur universim

$$
a_{2}=b_{2}-3 b_{3} \cdot e+\ldots=\partial_{c}^{2} B(e),
$$

$$
(-1)^{n} a_{n}=\boldsymbol{B}_{n}(e)=\underset{c}{\partial^{n}} \boldsymbol{B}_{(e)}
$$

Similiter aequationes

$$
\begin{aligned}
& \text { 1. } b=a_{0}+e a_{1}+e(e-1) a_{2}+e(e-1) \cdot(e-2) \cdot a_{3}+\ldots=A(e) \text {, } \\
& \text { 2. } b_{1}=a_{1}+2 a_{2} e+3 a_{3} e(e-1)+\ldots \ldots \ldots \ldots \ldots=\Delta A(e) \text {, } \\
& \text { 3. } b_{2}=a_{2}+3 e a_{3}+\ldots \ldots \ldots \ldots \ldots \ldots \ldots=\frac{1}{2} \Delta^{2} A(e) \\
& \text { etc. }
\end{aligned}
$$

ponendo $\boldsymbol{X}=x^{e}$, resolvuntur, etc. innumerae aliae.

Facto scilicet $\boldsymbol{Z}=x^{-e}$, erit ex modo demonstratis

$$
a_{0}=\left(b^{n} \partial\right)\left(x^{-e}\right)=x^{-e} b_{0}-e b_{1} x^{-1-e}+e(e+1) b_{2} x^{-2-e}-\ldots+\ldots
$$

seu, posito

$$
x=1, a_{0}=b_{0}-e b_{1}+e(e+1) b_{2}-e(e+1)(e+2) b_{3}+\ldots=B(e),
$$
et 


$$
\begin{gathered}
a_{1}=9\left(b^{n} \partial\right)\left(X^{-e}\right)=b_{1}-2 e b_{2}+3 e(e+1) b_{3}-4 . e(e+1) \cdot(e+2) \cdot b_{4}-\ldots . \\
={ }^{\prime} \Delta B(e), \\
a_{2}=b_{2}-3 e b_{3}+6 e(e+1) b_{4}-\text { etc. }=+\frac{1}{2}^{\prime} \Delta^{2} B(e), \text { etc. } \\
\text { (si } \Delta e=-1) .
\end{gathered}
$$

Ex his exemplis, quae facile directe demonstrantur, videre licet, theorema nostrum etiam ad valorem $n=\infty$ extendi.

\section{Fragmentum III.}

De aequalibus aequationum lineariter differentialium $(\stackrel{n}{a} \partial) y=0$ radicibus.

Quoniam ignotae hujusmodi aequationis ope determinandae non nisi functiones sunt, aequales sane habendae sunt radices, quae eadem functione exprimuntur. Olim ideo credidimus, alterutra inventa radice $y_{0}=f x$, tum aliam $y_{1}$, quae ipsius aequalis sit naturae, per eandem functionem $f x$ simplicissime (ex. gr. per $y_{1}=f(g x)$, existente $g x$ functione nota simpliciore, imo hujusmodi, ut $g(g x)=x$, vel $g^{n} x=x$ sit) exhiberi. Jam vero ex nostra theoria tagmatica demonstramus, radices aequales per $x^{m} \cdot f x$, existente $f x$ ipsarum simplicissima, explicari.

Sint enim $y_{0}=f x$, atque $y_{1}=x^{m} \cdot f x$, seu $y_{1}=x^{m} \cdot y_{0}$, radices aequationis $(\stackrel{n}{a} \partial) y=0\left(=a_{0} y+a_{1} \partial y+a_{2} \partial^{2} y+\ldots\right)$; eritque $(\stackrel{n}{a} \partial) y_{0}=0$, atque $(\stackrel{n}{a} \partial) y_{1}=(\stackrel{n}{a} \partial)\left(x^{m} y_{0}\right)=0$.

Est vero

- $\quad a^{n} \dot{\partial}\left(x^{m} y_{0}\right)=(\stackrel{n}{\partial} \partial) y_{0} \cdot x^{m}+\vartheta(\stackrel{n}{a} \partial) y_{0} \cdot m x^{m-1}+\frac{1}{2} \vartheta^{2}\left(a^{n} \partial\right) y_{0} \cdot m(m-1) \cdot x^{m-2}$

$$
+\ldots . g^{r}(\stackrel{n}{a} \partial) y_{0} \cdot m_{r} x^{m-r}+\ldots . .
$$

ubi quidem omnes termini aderunt, si $m>n$ fuerint; sin vero $m<n$ sit et integer, tum certe termini ultími, inde ab $r=m+1$ usque ad $r=n$, ultro evanescent. Ut igitur aequationi $(\stackrel{n}{a} \partial) y_{1}=0$ satisfiat, nihil restat, cum praeterea $(\stackrel{n}{a} \partial) y_{0}=0$ sit, nisi ut ponamus seorsim:

$$
\vartheta(\stackrel{n}{a} \partial) y_{0}=0, \quad \vartheta^{2}\left(a^{n} \partial\right) y_{0}=0, \quad \vartheta^{3}\left(a^{n} \partial\right) y_{0}=0 \text { etc. }
$$

usque ad $\vartheta^{m}(a) y_{0}=0$; quae quidem aequationes mutuum coëfficientium habitum, qualis ad $m+1$ radices aequales producendas requiratur, determinant. Patet enim, harum aequationum ope fore $(\stackrel{n}{a} \partial)\left(x^{r} y_{0}\right)=0$, dummodo numerus integer $r=$ vel $<m$ sit; ideoque aequationem $(\stackrel{n}{a} \partial) y_{0}=0$, seu 
$\boldsymbol{A}=0$, siquidem fuerint $\boldsymbol{\vartheta} \boldsymbol{A}=0, \vartheta^{2} \boldsymbol{A}=0, \vartheta^{3} \boldsymbol{A}=0, \ldots, \vartheta^{m} \boldsymbol{A}=0$; his gaudere $m+1$ radicibus $y_{0}, x y_{0}, x^{2} y_{0}, x^{3} y_{0}, \ldots x^{m} y_{0}$, quae sane aequales appellari possunt, cum simplicissime per eandem functionem $y_{0}=f x$ aeque exprimantur, nempe sub forma $\left(a+a_{1} x+a_{2} x^{2}+\ldots+a_{m} x^{m}\right) f x$, praetereaque vulgaris analysis, cujus ope radix $y_{1}$ per $y_{0}$ determinatur, si constantia certo modo accipiantur, $y_{1}=y_{0}$ const. suppeditet, ut in exemplo videre licet.

Ex. Ponamus $n=2$, seu $(\stackrel{2}{a} \partial) y=0$ et $\vartheta(\stackrel{2}{a} \partial) y=0$; id est

atque

$$
a_{0} y+2 a_{1} \partial y+a_{2} \partial^{2} y=0=A
$$

$$
a_{1} y+a_{2} \partial y=0=B
$$

(ubi commoditatis causa $2 a_{1}$ loco $a_{1}$ posuimus).

Erit igitur

atque

$$
\boldsymbol{A}-\partial \boldsymbol{B}=\left(a_{0}-\partial a_{1}\right) y+\left(a_{1}-\partial a_{2}\right) \partial y=0
$$

indeque aequalitatis conditio:

$$
-\frac{\partial y}{y}=\frac{a_{1}}{a_{2}}=\frac{a_{0}-\partial a_{1}}{a_{1}-\partial a_{2}}
$$

$$
\left(a_{0}-\partial a_{1}\right) a_{2}=a_{1}\left(a_{1}-\partial a_{2}\right)
$$

seu

$$
a_{0} \cdot a_{2}=a_{1}^{2}+a_{2} \partial a_{1}-a_{1} \partial a_{2}
$$

seu etiam simplicius $a_{0}=a_{1}^{2}+\partial a_{1}$, siquidem $a_{2}=1$, ut semper licet, effectum sit.

Haec igitur aequatio $\left(a_{1}^{2}+\partial a_{1}\right) y+2 a_{1} \partial y+\partial^{2} y=0$, aequalibus gaudet radicibus. Perquisita enim altera $y_{0}$ ex aequatione

$$
\frac{\partial y_{0}}{y_{0}}=-a_{1}
$$

quae $y_{0}=\beta^{-a_{1}} \partial x$ suppeditat, alteram $y_{1}$ vulgari via quaeramus. Terminis igitur ultimis ex aequationibus

$$
\text { et } \begin{aligned}
\partial^{2} y_{0}+2 a_{1} \partial y_{0}+\left(a_{1}^{2}+\partial a_{1}\right) y_{0} & =0 \\
\partial^{2} y_{1}+2 a_{1} \partial y_{1}+\left(a_{1}^{2}+\partial a_{1}\right) y_{1} & =0
\end{aligned}
$$

exterminatis, oritur $y_{1} \partial^{2} y_{0}-y_{10} \partial^{2} y_{1}+2 a_{1}\left(y_{1} \partial y_{0}-y_{0} \partial y_{1}\right)=0$ seu $\partial q+2 a_{1} \cdot q=0$, posito scilicet $q=y_{1} \cdot \partial y_{0}-y_{0} \partial y_{1}$. Integrando igitur obtinetur $L q=-2 \int a_{1} d x$ seu

$$
y_{1} \partial y_{0}-y_{0} \partial y_{1}=\beta^{-2 \int a_{1} d x}=c \cdot y_{0}^{2} ; \text { h. e. } \partial\left(\frac{y_{1}}{y_{0}}\right)=c
$$

iterumque exhinc $y_{1}=(a+x c) \cdot y_{0}$. Sin vero accipimus $c=0$; erit $\frac{\partial y_{1}}{y}$ 
$=\frac{\partial y_{0}}{y_{0}}$, ideoque $y_{1}=a \cdot y_{0}$. At radices $y_{0}$ et $a y_{0}$, ratione completae solutionis habita, haud diversae sunt censendae. Istius igitur aequationis solutio completa est $y=(\boldsymbol{A}+\boldsymbol{B} x) \beta^{-\int a_{1} \partial x}$.

Simile quid in altioribus gradibus observare licet. Exhinc igitur sequentia colliguntur theoremata:

$S i$ aliqua aequationis $\stackrel{n}{(a} \partial) y=0$, radix $y_{0}$ ejusmodi sit, ut tagma primum $\vartheta(\stackrel{n}{a} \partial) y_{0}=0$ sit, altera radix erit $x \cdot y_{0}$, et si praeterea $\vartheta^{2}\left(a^{n} \partial\right) y_{0}$ $=0$ fuerit, accedet radix $x^{2} y_{0}$, generatimque tot aderunt radices aequales, seu formae $x^{m} y_{0}$, quot aequationes, nempe ipsa tagmatica atque hujus derivatue.

Sic, si data fuerit aequatio tertii ordinis $\left(\dot{a}^{3} \partial\right) y=0$ seu $(A=) a_{0} y$ $+3 a_{1} \partial y+3 a_{2} \partial^{2} y+a_{3} \partial^{3} y=0$, simulque fuerit ( $\frac{1}{3} \vartheta A \Rightarrow a_{1} y+2 a_{2} \partial y+$ $a_{3} \partial^{2} y=0$, seu si tagma primum evanuerit, erit tum $\left(A \Rightarrow a_{0} y+3 a_{1} \partial y\right.$ $+3 a_{2} \partial^{2} y+a_{3} \partial^{3} y=0$, tum differentiando $\left(\frac{1}{3} \partial \mathcal{A} A\right) \partial a_{1} \cdot y+\left(a_{1}+2 \partial a_{2}\right) \partial y$ $+\left(2 a_{2}+\partial a_{3}\right) \partial^{2} y+a_{3} \partial^{3} y=0$ ideoque $\partial^{3} y$ eliminando

Est vero et

$$
\left(a_{0}-\partial a_{1}\right) \cdot y+2\left(a_{1}-\partial a_{2}\right) \partial y+\left(a_{2}-\partial a_{3}\right) \partial^{2} y=0 .
$$

ideoque ipso $\partial^{2} y$ ejecto,

$$
a_{1} y+2 a_{2} \partial y+a_{3} \partial^{2} y=0
$$

$0=\left(\left(a_{0}-\partial a_{1}\right) \cdot a_{3}-a_{1} \cdot\left(a_{2}-\partial a_{3}\right)\right) y+2 \cdot\left(\left(a_{1}-\partial a_{2}\right) a_{3}-a_{2} \cdot\left(a_{2}-\partial a_{3}\right)\right) \partial y \cdot$ Cujus jam aequationis ope functio $y$ facile determinatur, eaque aequalis est radix. Praeterea si $a_{3}=1$ efficitur, ut licet, ista aequatio simplicior

$$
0=\left(a_{0}-\partial a_{1}-a_{1} \cdot a_{2}\right) y+2\left(a_{1}-\partial a_{2}-a_{2}^{2}\right) \partial y
$$

evadit. Haec vero differentiata aequationem

$$
\begin{aligned}
0=\left(\partial a_{0}-\partial^{2} a_{1}-\partial\left(a_{1} a_{2}\right)\right. & y+\left(a_{0}-\partial a_{1}-a_{1} a_{2}+2 \partial a_{1}-2 \partial^{2} a_{2}-2^{2} \cdot a_{2} \partial a_{2}\right) \partial y \\
& +2\left(a_{1}-\partial a_{2}-a_{2}^{2}\right) \partial^{2} y
\end{aligned}
$$

praebet. Hac vero novà aequatione cum antiqua illa

$$
0=2\left(a_{1}-\partial a_{2}-a_{2}^{2}\right)\left(a_{1} y+2 a_{2} \partial y+\partial^{2} y\right)
$$

comparata aliam quoque hanc suppeditat aequationem

$$
\begin{gathered}
0=\left(\partial a_{0}-\partial^{2} a_{1}-a_{2} \partial a_{1}+a_{1} \partial a_{2}-2 a_{1}^{2}+2 a_{1} a_{2}^{3}\right) y \\
+\left(\partial a_{1}-2 \partial^{2} a_{2}-5 a_{1} a_{2}+4 a_{2}^{3}\right) d y
\end{gathered}
$$

quae etiam $\frac{\partial y}{y}$ determinat.

Utrosque igitur ipsius $d y: y$ valores comparando conditionem, ut aequatio proposita aequales habeat radices duas, necessariam elicies, idem- 
que in aequatione cujusvis ordinis simili omnino modo facile efficies. Hinc vero alterum elicimus theorema:

Aequationem differentialem ordinis cujusvis $\left(n^{o}\right)$ nempe $\left.\stackrel{n}{(a} \partial\right) y=0$, in qua aliqua de causa radicum aequalium par unicum ponere liceat, semper methodo Bernoulliana esse solubilem; quem in finem ipsius tagma primum $\vartheta(\stackrel{n}{a} \partial) y=0$ datae jungendum est, et derivata altiora excutienda, donec $\partial y: y$ in coëfficientibus datis exhibeatur. Sin vero plura aequalium paria adfuerint, prius subsistendum est, ex. gr. ad $\stackrel{2}{(a} \partial) y=0$, si duo sunt. Inde et simul rei verae conditionem necessariam elicies. Ex. gr. Si $\left(a^{2} \partial\right) y=0$ seu $a_{0} y+2 a_{1} \partial y+a_{2} \partial^{2} y=0$, et coëficientes $a_{0}, a_{1}, a_{2}$ constantes fuerint, erit in casu aequalium radicum $a_{1} y+a_{2} \partial y=0$, ideoque si $a_{1}=-\alpha$. $a_{2}$ ponitur, $\frac{d y}{y}=a d x$, et integrando $L y=a x+L C$ seu inverse

$$
y=\mathbf{T}(\alpha x+\mathbf{L} \boldsymbol{C})=C \cdot \mathbf{T}(\alpha x) \text {. * })
$$

Hujus vero solutionis conditio jam antea explioata est $\frac{a_{1}}{a_{2}}=\frac{a_{0}-\partial a_{1}}{a_{1}-\frac{\partial}{\partial a_{2}}}$, seu $a_{1}^{2}=a_{0} a_{2}$, id quod rei notissimae de indole radicum aequationis algebraicae $a^{2} \partial=0$ seu $a_{0}+2 a_{1} \partial+a_{2} \partial^{2}=0$ optimi, convenit.

Nec diffitemur, nos, cum, solutionis conatubus infinitis frustra susceptis, tandem persuasi fuerimus, has aequationes differentiales nullam quadraturae simplicis ope solutionem universalem admittere, ideoque de harum theoria conscribenda meditandum esse, ex ipsa hac analogia aequationis differentialis et algebraicae, quae saltem in casu coëfficientium constantium in dubium haud vocari potest, varia indicia disquisitionem adjuvantia hausisse. Universim enim ponendo $y=\mathbf{T}(\alpha x)$, aequatio $(\stackrel{n}{a} \partial) y=0$ coëfficientibus constantibus gaudens ad aequationem $(\tilde{a})^{0} \alpha=0$ ducit, et utraque non modo aequali radicum numero sed et simili indole gaudent, aequalibus utriusque radicibus sibi invicem concomitantibus. At si $\left({ }_{a}^{n}\right)^{\prime \prime} \alpha=0$ aequales habet radices, valet etiam aequatio $\partial_{\alpha}(\stackrel{n}{a})^{0}=0$ seu $a_{1}+2 a_{2} \alpha+3 a_{3} \alpha^{2}+\ldots=0$, quae et bre$\operatorname{viter}\left(\stackrel{n_{1}}{a}\right) \alpha=0$ scribi potest. Sin vero similem ipsius $(\stackrel{n}{a} \partial) y=0$ habitum

*) Duce cel. Laplace functiones inversas litteram initialem functionis invertendo designamus. Vulgaris igitur functio exponentialis $\beta^{x}$ nobis est $\mathbf{I} x$, ex theoria iterationis vero per $\mathrm{L}^{-1} x$ indicatur. 
volumus, ideoque $\vartheta(\stackrel{n}{a} \partial) y=0$ ponimus, seu $a_{1} y+2 a_{2} \partial y+3 a_{3} \partial^{2} y+\ldots=0$, patet et hanc aequationem fore $\left(a^{n_{1}}\right) \partial y=0$; et, substitutione $y=\mathbf{T}(\alpha x)$ (seu $=\beta^{\alpha x}$ ) effecta, inde hanc oriri aequationem $\left(\stackrel{n_{1}}{a}\right) \alpha=0$, seu eandem ac ex conditione radicum aequalium in aequatione algebraica huic differentiali respondente. Inde justam esse functionalium radicum aequalium notionem, facile argues. Idem et aliunde comprobari potest.

Hac radicum aequalium doctrina in solutionibus aequationum particulariter differentialium adaptandis interdum magni erit usus. Ut si inventa fuerit huiusınodi solutio: $z=\frac{\left(n^{\prime} \partial\right) \varphi x}{\left(r^{\prime} \partial\right) \psi y}$, reique natura desideraverit, ut functiones $\phi x$ et $\psi y$, (quae universim arbitrariae erant) radices sint aequationum lineariter particularium $\left(n^{\prime} \partial\right) X=0$ et $\left(r^{\prime} \partial\right) \boldsymbol{Y}=0$, (ubi coëfficientes $n_{1}, n_{1}, n_{2}$ et, $r_{11}, r_{1}, r_{2}$ et tum $y$ tum $x$ continere possunt), is(a solutio valorem ipsius $\boldsymbol{Z}$ sub forma indeterminata .0 .0 , vix nisi tagmatice definieuda, praebebit. Exemplo tibi sit casus, quo $\boldsymbol{X}$ et $\boldsymbol{Y}$ functiones sint Ellipticae notissimae $\boldsymbol{E}^{\prime}$ et $\boldsymbol{H}^{\prime \prime}$. Pergralum foret, si quis, quid calculus Variationum ad hunc nodum dissolvendum valeat, edocere vellet.

\section{Fragmentum IV.}

De solutione acquationis linearis, $\left(n^{\prime} \partial\right) u=0$.

Forma $\left(n^{\prime} \partial\right) y=n_{0} y+n_{1} \partial y+n_{2} \partial^{2} y+\ldots .+n_{n} \partial^{n} y$ aperte distributivae est indolis seu ejusmodi, ut $\left(n^{\prime} \partial\right)(y+z)=\left(n^{\prime} \partial\right) y+\left(n^{\prime} \partial\right) z$ sit. Productum vero $z y$ ipsa, ut in Fr. I. docuimus, hunc in modum dissolvit:

$$
\begin{aligned}
& \left(n^{\prime} \partial\right)(z y)=\left(n^{\prime} \partial\right) z \cdot y+\vartheta\left(n^{\prime} \partial\right) z . \partial y+\frac{\vartheta^{2}}{1.2}\left(n^{\prime} \partial\right) z . \partial^{2} y+\ldots \\
& \ldots+\frac{\vartheta^{r}}{1.2 .3 \ldots . . r}\left(n^{\prime} \partial\right) z \cdot \partial^{r} y+\ldots .+n_{n} \cdot z \cdot \partial^{n} y \text {. }
\end{aligned}
$$

Jam vero proposita aequatione lineariter differentiali $\left(\dot{n}^{\prime} \partial\right) u=0$, ipsa universim solutu erit difficillima, nisi aliquo modo radicem particularem $u_{0}$ iuvenire liceat. Jam vero hujus evolutionem, quam etiam convergentem efficere liceat, proponendi est animus. Facto itaque $u=a+y,\left(\dot{n}^{\prime} \partial\right) y=$ $y-\left(n^{\prime} \partial\right) y$ atque $\left(\dot{n}^{\prime} \partial\right) a=q$, erit $\left(\dot{n}^{\prime} \partial\right) y=-\left(\dot{n}^{\prime} \partial\right) a=q, \operatorname{seu}\left(n^{\prime} \partial\right) y+q=y$, quae jam est aequatio solvenda. Quem in finem iteratis vicibus ipsam operationi tagmaticae $\left(n^{\prime} \partial\right)$ subjiciamas, eritque

Crelle's Journal f. d. M. Bd. XXV. Heft 1. 


$$
\begin{gathered}
\left(n^{\prime} \partial\right) y=\left(n^{\prime} \partial\right)\left(q+n^{\prime} \partial y\right)=\left(n^{\prime} \partial\right) q+\left(n^{\prime} \partial\right)\left(n^{\prime} \partial\right) y, \text { seu, si } \\
\left(n^{\prime} \partial\right)^{2} q=\left(n^{\prime} \partial\right)\left(n^{\prime} \partial\right) q, \\
y=q+\left(n^{\prime} \partial\right) q+\left(n^{\prime} \partial\right)^{2} y, \text { rursumque, si } \\
\left(n^{\prime} \partial\right)^{2}\left(n^{\prime} \partial\right) q=\left(n^{\prime} \partial\right)^{3} q, \quad\left(n^{\prime} \partial\right)^{2} y=\left(n^{\prime} \partial\right)^{2} q+\left(n^{\prime} \partial\right)^{3} y,
\end{gathered}
$$

ideoque, substituendo,

$$
y=q+\left(n^{\prime} \partial\right) q+\left(n^{\prime} \partial\right)^{2} q+\left(n^{\prime} \partial\right)^{3} y
$$

Apparet jam, ita continuando obtentum iri

$$
y=q+\left(n^{\prime} \partial\right) q+\left(n^{\prime} \partial\right)^{2} q+\left(n^{\prime} \partial\right)^{3} q+\ldots .+\left(n^{\prime} \partial\right)^{r} q+\left(n^{\prime} \partial\right)^{r+1} y .
$$

$\mathrm{Si}$ igitur termini hujus polynomii tandem magis magisque diminuerint, $r=\infty$ atque $\left(n^{\prime} \partial\right)^{r+1} y=0$ efficere licebit, eritque aequationis linearis $\left(n^{\prime} \partial\right) y+q$ $=y$ haec solutio

$$
y=q+\left(n^{\prime} \partial\right) q+\left(n^{\prime} \partial\right)^{2} q+\left(n^{\prime} \partial\right)^{3} q+\left(n^{\prime} \partial\right)^{4} q+\ldots,
$$

quae series singulares omnino est formae.

Hujus vero termini successive ex sese calculantur secundum formulam

$$
\left(\boldsymbol{n}^{\prime} \partial\right)^{r+1} \boldsymbol{q}=\left(\boldsymbol{n}^{\prime} \partial\right)\left(\boldsymbol{n}^{\prime} \partial\right)^{r} q
$$

quae valet, si jam inventum fuerit

$$
\begin{aligned}
& \left(\boldsymbol{n}^{\prime} \partial\right)^{r} q=\boldsymbol{R}, \text { erit }\left(\boldsymbol{n}^{\prime} \partial\right)^{r+1} q=\left(\boldsymbol{n}^{\prime} \partial\right) \boldsymbol{R} \text { seu } \\
& =n_{0} \boldsymbol{R}+n_{1} \boldsymbol{R}+n_{2} \partial^{2} \boldsymbol{R}+\ldots+n_{n} \partial^{n} \boldsymbol{R} .
\end{aligned}
$$

Ipsi omnes igitur finiti sunt, si $n$ numerus est finitus. Speciatim vero est ideoque erit

$$
\left(n^{\prime} \partial\right) q=n_{0} q+n_{1} \partial q+n_{2} \partial^{2} q+\ldots+n_{n} \partial^{n} q
$$

$$
\left(n^{\prime} \partial\right)^{2} q=\left(n^{\prime} \partial\right)\left(n_{0} q\right)+\left(n^{\prime} \partial\right)\left(n_{1} \partial q\right)+\ldots+\left(n^{\prime} \partial\right)\left(n_{1} \partial^{n} q\right)
$$

qui valor per theorema antea expositum ulterius ad formam $\Theta_{0} q+\Theta_{1} \partial q$ $+\Theta_{2} \partial^{2} q+\ldots$ explicari potest. Hanc viam igitur saepius calcando perveniemus ad hujusmodi valorem explicitum

$$
\boldsymbol{R}=\left(\boldsymbol{n}^{\prime} \partial\right)^{r} q=\Theta_{0}^{r} \cdot q+\Theta_{1}^{r} \cdot \partial q+\Theta_{2}^{r} \cdot \partial^{2} q+\Theta_{3}^{r} \cdot \partial^{3} q+\ldots .
$$

rursumque

$$
\begin{aligned}
\left(n^{\prime} \partial\right)^{r+1} q & =\Theta_{0}^{r+1} \cdot q+\Theta_{1}^{r+1} \partial q+\Theta_{2}^{r+1} \cdot \partial^{2} q+\ldots, \text { quod et } \\
& =\left(n^{\prime} \partial\right)\left(\Theta_{0}^{r} \cdot q\right)+\left(n^{\prime} \partial\right)\left(\Theta_{1}^{r} . \partial q\right)+\left(n^{\prime} \partial\right)\left(\Theta_{2}^{r} . \partial^{2} q\right)+\ldots
\end{aligned}
$$

Ulteriori igitur evolutione facta, comparatisque coëfficientibus ipsorum $q, \partial q, \partial^{2} q \ldots .$, erit

$$
\begin{aligned}
& \Theta_{0}^{r+1}=\left(n^{\prime} \partial\right) \Theta_{0}^{r}, \\
& \Theta_{1}^{r+1}=\left(n^{\prime} \partial\right) \Theta_{1}^{r}+\vartheta\left(n^{\prime} \partial\right) \Theta_{0}^{r}, \\
& \Theta_{2}^{r+1}=\left(n^{\prime} \partial\right) \Theta_{2}^{r}+\vartheta\left(n^{\prime} \partial\right) \Theta_{1}^{r}+\frac{1}{1.2} \vartheta^{2}\left(n^{\prime} \partial\right) \Theta_{0}^{r}, \\
& \Theta_{3}^{r+1}=\left(n^{\prime} \partial\right) \Theta_{3}^{r}+\vartheta\left(n^{\prime} \partial\right) \Theta_{2}^{r}+\frac{1}{1.2} \cdot \vartheta^{2}\left(n^{\prime} \partial\right) \Theta_{1}^{r}+\frac{1}{1.2 .3} \vartheta^{3}\left(n^{\prime} \partial\right) \Theta_{\bullet}^{r}, \\
& \text { etc. }
\end{aligned}
$$


Quarum formularum ope coëficientes $\Theta$ successive calculari possunt.

Jam vero si hos valores explicitos in solutionem antea inventam introducimus, hac hujusmodi formam nanciscetur

$$
\boldsymbol{y}=\boldsymbol{Q} q+\boldsymbol{Q}_{1} \cdot \partial q+\boldsymbol{Q}_{2} \cdot \partial^{2} q+\boldsymbol{Q}_{3} \cdot \partial^{3} q+\ldots+\boldsymbol{Q}_{\infty} . \partial^{\infty} q .
$$

Sin vero coëfficientes $Q_{0}, Q_{1}, Q_{2}$ etc. directe determinare vellemus, sufficienda foret haec ipsa series in locum ipsius $y$ in aequatione

$$
y=q+\left(n^{\prime} \partial\right) y,
$$

quo facto, comparationeque instituta hae obtinentur aequationes:

$$
\begin{array}{ll}
\text { 1. } & \boldsymbol{Q}_{0}=1+\left(n^{\prime} \partial\right) \boldsymbol{Q}_{0} \\
\text { 2. } & \boldsymbol{Q}_{1}=\left(n^{\prime} \partial\right) \boldsymbol{Q}_{0}, \\
\text { 3. } & \boldsymbol{Q}_{2}=\left(n^{\prime} \partial\right) \boldsymbol{Q}_{1}+\vartheta\left(n^{\prime} \partial\right) \boldsymbol{Q}_{0}, \\
\text { 4. } & \boldsymbol{Q}_{3}=\left(\boldsymbol{n}^{\prime} \partial\right) \boldsymbol{Q}_{2}+\vartheta\left(n^{\prime} \partial\right) \boldsymbol{Q}_{1}+\frac{1}{1.2} \cdot \vartheta^{2}\left(n^{\prime} \partial\right) \boldsymbol{Q}_{0}, \text { etc. }
\end{array}
$$

Jam vero aequatio (1.) aperte docet, quantitatem $\boldsymbol{Q}$ solutionem esse particularem seu radicem aequationis propositae $y=q+\left(n^{\prime} \partial\right) y$, ei casu adaptatim, quo $q=1$. Inde vero arguere licet evolutionem antecedentem justam fore (dummodo $q$ nullius aequationis finitae $\left(m^{\prime} \partial\right) q=0$ sit radix), tolliturque ita scrupulus ex positione $\left(n^{\prime} \partial\right)^{\infty} y=0$ oriundus. Sin vero ad finitum terminum subsistere tibi libuerit, sane valor ipsius $\left(n^{\prime} \partial\right)^{r+1} y$ aliquatenus calculandus erit, unicuique casu particulari adaptandus est $\varphi$ ita ut $\left(n^{\prime} \partial\right)^{r} q$ quam minimus evadet. Quod etiam ut problema maximi momenti est censendum, ideoque Geometrarum attentionem mereri videtur. Hoc tantummodo in praesenti observare lubet, functionem

$$
q=\boldsymbol{A} \beta^{\omega x} \text { seu } \int \boldsymbol{A}_{r} \beta^{\omega_{r} x}
$$

effici posse, existentibus $\omega$ vel $\omega_{r}$ quantitatibus quibusvis, quas ideo quam minimas accipias, ut $\partial^{\infty} q$ evanescat. Si enim proposita fuerit aequatio

seu

$$
u+v, \quad \partial u+v_{2} \partial^{2} u+\ldots .=0
$$

poniturque $u=a+y$, erit

$$
\left(\nu^{\prime} \partial\right) u=0
$$

$$
\left(\nu^{\prime} \partial\right) y+\left(\nu^{\prime} \partial\right) \grave{a}=0
$$

ideoque (nisi forte $\left(\nu^{\prime} \partial\right) a=0$, quo casu solutio particularis $u=a$ inventa est), erit

si jam

$$
\frac{q \cdot\left(v^{\prime} \partial\right) y}{\left(\nu^{\prime} \partial\right) a}+q=0 \text { et } y=q+\left(n^{\prime} \partial\right) y
$$

accipitur, seu

$$
q\left(\nu^{\prime} \partial\right) y=\left(\nu^{\prime} \partial\right) a \cdot\left(\left(n^{\prime} \partial\right) y-y\right)
$$




$$
\left(n^{\prime} \partial\right) y=y+q \cdot\left(\frac{y+\nu_{1} \partial y+\nu_{2} \partial^{2} y+\ldots . .}{a+\nu_{1} \partial a+\nu_{2} \partial^{2} a+\ldots .}\right)
$$

facitur. Duae igitur adsunt functiones arbitrariae $q$ et $a$, quarum illa sub forma quam diximus, optime sumitur, haec vero ita determinanda est ut $\left(n^{\prime} \partial\right)^{\infty} y$ evanescat. Commodius tamen inverso ordine limites integrandi ita determinabuntur, ut hoc locum habeat; deindeque, alio valore vel alia forma ipsius $a$ adhibita, novi invenientur limites, hocque iteretur, donec totus integrandi campus exhaustus sit.

Jam vero redeamus ad aequationes mixte differentiales, $\Theta$, quae ad quantitates $\Theta$ determinandas inservirent. Harum prima $\Theta_{0}^{r+1}=\left(n^{\prime} \partial\right) \Theta_{0}^{r}$ seu $\Theta_{0}^{r+1}=n_{0} \Theta_{0}^{r}+n_{1} \partial \Theta_{0}^{r}+n_{2} \partial^{2} \Theta_{0}^{r}+\ldots$. facile solvitur et suppeditat $\Theta_{0}^{r}=$ $\left(n^{\prime} \partial\right)^{r-1} t$, exsistente $t$ functione arbitraria. Quia vero heic $\Theta_{0}^{\prime}=n_{0}$, erit $t=n_{0}$ et $\Theta_{0}^{r}=\left(n^{\prime} \dot{\partial}\right)^{r-1} n_{0}$. Altera vero $\Theta_{1}^{r+1}=\left(n^{\prime} \partial\right) \Theta_{1}^{r}+\vartheta\left(n^{\prime} \partial\right) \Theta_{0}^{r}$, si breviter $\boldsymbol{N}$ pro $\left(n^{\prime} \partial\right)$ scribimus, hujusmodi gaudet solutione

$$
\Theta_{1}^{r}=N^{r+1} t_{0}+N^{r-2} \vartheta N t_{0}+N^{r-3} \vartheta N^{2} t_{0}+N^{r+4} \vartheta N^{3} t_{0}+\ldots .+\vartheta N^{r-1} t_{0} ;
$$

hac enim admissa erit

$$
\Theta_{1}^{r+1}=N^{r} t_{0}+N^{r-1} \vartheta N t_{0}+N^{r-2} \vartheta N^{2} t_{0}+\ldots .+N \vartheta N^{r-1} t_{0}+\vartheta N^{r} t_{0},
$$

quod aperte a $\Theta_{1}^{r}$ cum $\vartheta N \Theta_{1}^{r}$ differt, si $N^{r} t_{0}=N \Theta_{0}^{r}=N^{r} n_{0}$ seu $t_{0}=n_{0}$ ponitur. Praeterea cum $\Theta_{1}^{\prime}=n_{1}$ sit, et ex formula inventa $=t_{1}+\vartheta t_{0}=t_{1}$, (est enim $\vartheta t_{0}=\vartheta n_{0}=0$, quia hic terminus originem ducit a $\left(n^{\prime} \partial\right)^{0}\left(n_{0} q\right)$ $\left.=\left(n^{\prime} \partial\right)^{0} n_{0} \cdot q+\vartheta\left(n^{\prime} \partial\right)^{0} n_{0} . \partial q+\ldots=n_{0} q\right)$, erit hic $t_{1}=n_{1}$, ideoque

$$
\Theta_{1}^{r}=N^{r-1} n_{1}+\ldots .+N^{r-2} \vartheta N n_{0}+N^{r-3} \vartheta N^{2} n_{0}+N^{r-4} \vartheta N^{3} n_{0}+\ldots .
$$

ubi rursum $\left(n^{\prime} \partial\right)$ pro $N$ restituendum est. Aeque reliquae aequationes solventur, inveuienturque $\Theta_{2}^{r}, \Theta_{3}^{r}, \ldots \Theta_{\nu}^{r}$, tametsi sub forma satis ampla.

His igitur valoribus adhibitis erit radix tagmatica

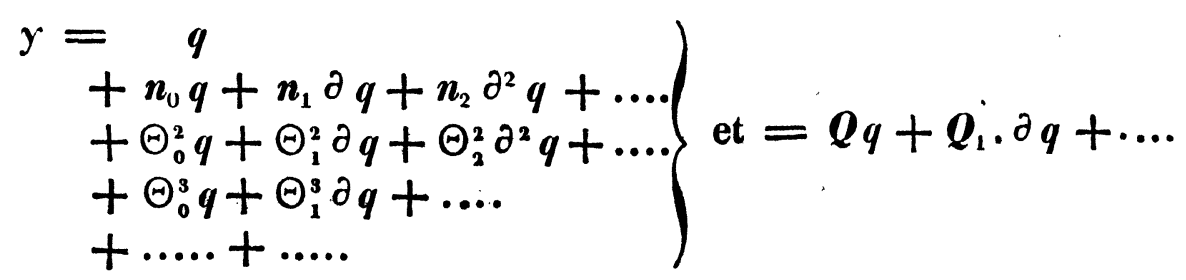

ideoque $\boldsymbol{Q}=1+n_{0}+\Theta_{0}^{2}+\Theta_{0}^{3}+\ldots .$. , seu

$$
\boldsymbol{Q}_{0}=1+n_{0}+\left(n^{\prime} \partial\right) n_{0}+\left(n^{\prime} \partial\right)^{2} n_{0}+\left(n^{\prime} \partial\right)^{3} n_{0}+\ldots .
$$

quae jam est solutio particularis aequationis tagmaticae

$$
y=1+n_{0} y+n_{1} \partial y+n_{2} \partial^{2} y+\ldots .+n_{n} \partial^{n} y, \text { seu } y=1+\left(n^{\prime} \partial\right) y \text {. }
$$


Probatio vero facillima est, si admittitur $\left(n^{\prime} \partial\right)^{\infty}, n_{0}=0$. Facto enim

$$
y=1+n_{0}+N n_{0}+N^{2} n_{0}+N^{3} n_{0}+\ldots .
$$

erit

$$
\boldsymbol{N} y=N\left(1+n_{0}+N n_{0}+\ldots\right)=N 1+N n_{0}+N^{2} n_{0}+N^{3} n_{0}+\ldots .
$$

quia $\boldsymbol{N}=\left(\boldsymbol{n}^{\prime} \partial\right)$ distributivum est, ideoque

seu

$$
1+N y=1+N+N n_{0}+N^{2} n_{0}+\ldots .
$$

quia

$$
1+N y=y
$$

$$
N_{1}=\left(n^{\prime} \partial\right) 1=n_{0} \cdot 1+n_{1} \partial_{1}+n_{2} \partial^{2} 1+=n_{0} .
$$

Haec vero solutio symbolice breviter ita representari potest:

$$
y=s+(1-N)^{-1} n_{0}, \text { seu } y=s+\left(1-\left(n^{\prime} \partial\right)\right)^{-1} n_{0} .
$$

Casus, quo $\left(n^{\prime} \partial\right) n_{0}=c=$ const. fuerit, facile solutionem finitam admittit. Quia enim $N n_{0}=c$, erit

$$
\begin{gathered}
N^{2} n_{0}=N c=n_{0} c+n_{1} \partial c+\ldots . .=n_{0} c, \quad N^{3} n_{0}=c N n_{0}=c^{2} \\
N^{4} n_{0}=N\left(c^{2}\right)=c^{2} n_{0}, \quad N^{3} n_{0}=c^{2} N n_{0}=c^{3} \text { etc. }
\end{gathered}
$$

ideoque

$$
y=1+n_{0}+c+n_{0} c+c^{2}+n_{0} c^{2}+c^{3}+\ldots=1+\left(n_{0}+c\right)\left(1+c+c^{2}+\ldots\right)
$$

seu

$$
y=1+\frac{n_{0}+c}{1-c}=\frac{1+n_{0}}{1-c}(\text { saltem si } c<1)
$$

Tum vero functiones $n_{0}, n_{1}, n_{2}$ etc. necessario ejusmodi sint, ut $\left(n^{\prime} \partial\right) n_{0}=c$, seu $c=n_{0}^{2}+n_{1} \partial n_{0}+n_{2} \cdot \partial^{2} n_{10}+n_{3} \partial^{3} n_{0}+\ldots+n_{n} \partial^{n} n_{0}$ sit.

Berolini 1833, Septb. 